\title{
Dominique Chancé, Patrick Chamoiseau, écrivain postcolonial et baroque
}

\section{Luigia Pattano}

\section{Q OpenEdition}

\section{Journals}

\section{Édition électronique}

URL : http://journals.openedition.org/studifrancesi/6136

DOI : 10.4000/studifrancesi.6136

ISSN : 2421-5856

Éditeur

Rosenberg \& Sellier

\section{Édition imprimée}

Date de publication : 1 mai 2011

Pagination : 227-229

ISSN : 0039-2944

\section{Référence électronique}

Luigia Pattano, «Dominique Chancé, Patrick Chamoiseau, écrivain postcolonial et baroque », Studi

Francesi [En ligne], 163 (LV | I) | 2011, mis en ligne le 30 novembre 2015, consulté le 12 janvier 2021.

URL : http://journals.openedition.org/studifrancesi/6136; DOI : https://doi.org/10.4000/studifrancesi. 6136

Ce document a été généré automatiquement le 12 janvier 2021.

\section{(c) 9 (i) $\Theta$}

Studi Francesi è distribuita con Licenza Creative Commons Attribuzione - Non commerciale - Non opere derivate 4.0 Internazionale. 


\title{
Dominique Chancé, Patrick Chamoiseau, écrivain postcolonial et baroque
}

\author{
Luigia Pattano
}

\section{RÉFÉRENCE}

DOMINIQUE CHANCÉ, Patrick Chamoiseau, écrivain postcolonial et baroque, Paris, Honoré

Champion, 2010, pp. 387.

1 Parmi les sujets les plus dramatiques et les plus fascinants de la sociologie des départements français des Antilles, la question nominale renvoyant à la violence symbolique de l'attribution du premier patronyme à la grande majorité de la population, qui marqua le début de l'époque coloniale post-esclavagiste, revient constamment dans plusieurs récits d'auteurs guadeloupéens et martiniquais contemporains. Visiblement fascinée par le poids symbolique d'une telle problématique collective dans une œuvre aussi foisonnante que celle de Patrick Chamoiseau, Dominique Chancé inaugure son dernier essai par une réflexion sur les patronymes qui la conduit à assumer cette question comme métaphore structurante de son volume consacré au célèbre romancier martiniquais. Cependant, elle ne s'attache pas, comme on pourrait le penser, à réfléchir sur les noms ou les actes de nommer présents dans l'archipel romanesque de l'écrivain: c'est plutôt les noms, surnoms et sobriquets du personnage-narrateur Patrick Chamoiseau qui l'interpellent car ils recouvriraient «des fonctions et des caractéristiques différentes dans l'œuvre» (p. 14). Un rapide coup d'œil à la table des matières montre que les noms et les attributs dont l'écrivain affuble son alter ego fictif sont, en effet, associés par Chancé à des moments différents et complémentaires de sa poétique. Quatre chapitres sur cinq portent dans le titre cette idée: «L"Oiseau de Cham", la trace africaine» (chapitre 1), «Chamzibié, éloge de la créolité» (chapitre 2), «Chamzibié et Chamoiseau: le marqueur de paroles» (chapitre 3) et «Chamoiseau écrivain baroque» (chapitre 4). La progression des chapitres allant de 
pair avec le cheminement de l'œuvre étudiée dans une optique chronologique, on comprend vite que Dominique Chancé lit la production littéraire de Chamoiseau comme l'expression d'une poétique à plusieurs facettes et à plusieurs stades, atteignant son plein achèvement dans l'ouvrage qu'elle considère comme étant le plus complexement baroque, ou le plus baroquement complexe: Biblique des derniers gestes.

2 Si on peut tout à fait apprécier l'originalité de cette lecture nourrie en apparence d'une symbolique onomastique, l'exploration des chapitres mentionnés ainsi que du dernier («Merveille et mélancolie») nous laisse un peu perplexes par rapport à la place marginale que Chancé réserve à la question évoquée en ouverture, à savoir la centralité du nom dans l'œuvre chamoisienne, introduite, pourtant, dans le second chapitre liminaire («Chamoiseau, Chamzibié, Oiseau de Cham, "l'écrivain au curieux nom d'oiseau"») en des termes si nets: "Toute l'écriture de Chamoiseau pourrait se révéler dans ce travail du nom, comme travail du nom. Le nom révèle d'abord ce qu'il y a de commun (oiseau, gibier) et de collectif (oiseau de Cham), avant de s'assumer dans sa singularité. Le nom propre n'est pas ou plus seulement un donné, un inné ou acquis familial et individuel, il résulte d'un détour, de l'appropriation d'une histoire et d'un langage collectifs, avant de devenir patronyme» (p. 18-19). Malgré une telle emphase, il semblerait plutôt que Chancé laisse de côté cette question au bénéfice d'une lecture poétique et idéologique de l'œuvre narrative de Chamoiseau en s'appuyant sur les textes théoriques de celui-ci (Éloge de la créolité et Lettres créoles), et sur ses récits autobiographiques (les trois tomes d'Une enfance créole et Écrire en pays dominé). Une autre source théorique importante pour élucider la poétique de Chamoiseau est représentée par quelques textes d'Édouard Glissant, parmi lesquels Le Discours antillais, Poétique de la Relation et Traité du Tout-Monde.

Intitulé donc "L'"Oiseau de Cham", la trace africaine», le premier chapitre de cet essai est consacré à ce que Chancé nomme la «négritude enracinée» ou «américaine» (p. 39) de l'auteur, qui consisterait en un projet esthétique (et éthique) visant à associer l'attachement à l'Afrique défendu par Césaire à l'exploration des particularités géographiques, linguistiques et culturelles de l'univers antillais. Cette tendance qu'elle repère dans la pièce de "théâtre conté» de Chamoiseau, Manman Dlo et la fée Carabosse (1982), serait également présente dans les trois premiers romans de l'auteur et attestée par la préférence accordée à la réalité quotidienne de la partie la plus faible de la population martiniquaise, nommée «Nègre» par Chancé d'après Chamoiseau. C'est donc par là, par cette attention portée aux Nègres de Martinique, que le romancier exprimerait sa volonté de reterritorialiser la trace africaine qui constitue un patrimoine génétique et culturel fondamental et indéniable pour ceux qu'il appelle «créoles». Si ces hypothèses, ces interprétations, montrent parfois quelques faiblesses car Chancé attribue à Chamoiseau à la fois une négritude, une créolité et une antillanité sans bien circonscrire et justifier ses choix terminologiques, il faut reconnaître que l'analyse ponctuelle des ouvrages littéraires est souvent très pertinente, voire précieuse.

Des critiques similaires pourraient être réitérées pour le deuxième chapitre, dont le but explicite est «d'essayer de saisir ce que c'est qu'être créole dans l'œuvre de Patrick Chamoiseau et à partir de la posture d'un certain Chamzibié, figure de fiction qui représente en partie l'auteur, dans l'univers de la créolité» (p. 76). La référence théorique est visiblement le manifeste créoliste: Éloge de la créolité. La démarche consiste à lire la présence de figures auctoriales portant le même nom que l'auteur dans 
l'œuvre chamoisienne et d'en tirer des implications poétiques et politiques. Lorsqu'elle se penche sur la foule de personnages populaires qui habitent les romans de l'auteur (y compris Chamoiseau lui-même), Chancé s'étonne de remarquer l'absence textuelle de termes socio-politiques qu'elle définit comme européens ou occidentaux, tels que "prolétaires», «ouvriers», "peuple», et elle en déduit une «inadéquation entre les catégories sociopolitiques décrivant les sociétés modernes et la créolité» (p. 84). Elle pousse plus loin son raisonnement: «la créolité est la formulation d'une inadéquation entre les schémas sociologiques occidentaux et le monde qu'elle se propose de décrire. Elle s'énonce comme une donnée culturelle (ou peut-être anthropologique, ethnologique ou tout simplement humaine) parce que la sociologie politique occidentale ne convient pas» (p. 84). Chancé reprend certains arguments postcoloniaux sur l'inadéquation du langage occidental en contexte colonial ou néocolonial en s'appuyant sur des exemples très parlants tirés des textes narratifs de Chamoiseau. Très pertinente nous semble, en particulier, l'analyse de l'idéologie mulâtre mise en scène par Patrick Chamoiseau et Raphaël Confiant ainsi que la mise en évidence d'une différence majeure entre les œuvres de ces deux auteurs. Si, dans la production du second, toute relation entre individus appartenant à des groupes raciaux différents implique toujours un certain degré de conflictualité, celle du premier ne sous-tend pas nécessairement cette violence.

5 Le chapitre "Chamzibié et Chamoiseau: le "marqueur de paroles"» aborde à la fois des questions de poétique et les mises en scène linguistiques dans les romans de Chamoiseau. Signalons, en particulier, deux paragraphes («Diglossie et stratégies linguistiques» et «De la diglossie à l'hybridité») qui proposent une réflexion autour du thème des langues et de son évolution dans l'œuvre du romancier martiniquais. Associant la diglossie à la réflexion sur la/les langue/s d'écriture, Dominique Chancé montre de façon très convaincante comment, dans le premier roman de l'auteur, l'alternance entre français et créole et la pratique scripturale de traduction sur le plan de l'énonciation répondent aux exigences d'une poétique qui évolue au fil du temps. Si Chronique affichait le désir de faire passer le créole en français dans «un rapport linguistique de traduction», Solibo Magnifique envisage la possibilité d'«un rapport culturel par trasmission» car, dans la fiction romanesque, le récit a été reconstruit à posteriori par un personnage qui se dit «marqueur de paroles» professionnel. À partir de Texaco, la question de la diglossie sera par contre dépassée par l'élaboration d'un français hybride, ce qui implique une pratique, et une conception, de la diversité comme tissage et non plus comme dichotomie.

6 La présence du baroque présentée dans le titre comme l'une des marques de Patrick Chamoiseau n'est traitée longuement que dans les deux derniers chapitres. Après avoir analysé la problématique de l'écriture qui traverserait, dans une "quête de symbolisation", toute l'œuvre de l'auteur, le quatrième chapitre corrige une première hypothèse de lecture du romanesque chamoisien et affirme que Chamoiseau n'a jamais abandonné la créolité, mais qu'il l'a enrichie en l'ouvrant à «une créolisation universelle, élémentaire, [...] de Relations infinies [...] et imprévisibles. L'identité ouverte, non définie, de la créolité, s'est complexifiée en identités incertaines, novatrices, engendrant des créations et des créatures surprenantes.» (p. 234). Dans ces propos, non seulement créolité et créolisation finissent par se mélanger, mais le baroque intervient comme une évidence dans ses références américaines sans que le problème de la définition du terme, et de son historique, ne soit posé. Les conclusions de Dominique Chancé peuvent se résumer de la façon suivante: l'écriture de 
Chamoiseau est axée sur l'«épanouissement d'un baroque implicite mais d'abord contenu, de Chronique des sept misères à Biblique des derniers gestes» (p. 12). La question n'est donc plus de savoir s'il y a du baroque chez Chamoiseau et à partir de quel roman, puisque Patrick Chamoiseau est un «écrivain postcolonial et baroque», mais de savoir comment le baroque s'articule dans l'ensemble de l'œuvre, et notamment par rapport à certains thèmes comme la dimension collective et individuelle (nous/je), le dialogisme, l'érotisme et l'altérité de la femme. Nous nous limitons ici à mentionner les conclusions de la première interrogation, que Chancé aborde de façon plutôt brillante. Elle affirme en effet que l'œuvre de Chamoiseau témoigne d'un passage du nous, «expression d'une communauté factice et illusoire qui fonde une identité idéologique, ponctuelle» dans Chronique, au ««je» symbolique et collectif auquel chacun peut s'identifier sans perdre sa singularité» (p. 245) dans L'esclave vieil homme. Au sein d'une telle trajectoire, le dernier roman analysé, Biblique des derniers gestes, est lu comme une synthèse de tous les procédés mis en place par l'auteur dans les ouvrages antécédents, comme un approfondissement et en même temps une expansion.

7 «Merveille et mélancolie», cinquième et dernier chapitre de l'ouvrage, débute sur une tentative de périodisation de l'œuvre de Chamoiseau, qui est divisée en deux phases, et sur le rapprochement entre le cheminement du romancier et celui d'Édouard Glissant, véritable père spirituel du premier. Si pour ce dernier la démarcation se situe en 1981, année de la publication du Discours antillais, pour Chamoiseau, elle se trouve dans deux ouvrages publiés en 1997: Écrire en pays dominé et L'esclave vieil homme et le molosse. Cette année marquerait pour Chamoiseau le passage du merveilleux créole des premiers romans, plus proche du réalisme merveilleux d'Alexis que du real maravilloso de Carpentier, à la mélancolie et à la merveille baroques des derniers ouvrages. La pensée de Chamoiseau aurait donc viré en 1997 vers une «conception postmoderne dans laquelle triomphe la pensée du baroque et de la complexité», dont témoigne également le cheminement de la trilogie autobiographique Une enfance créole (p. 308).

Après avoir essayé de schématiser l'œuvre de Patrick Chamoiseau, Chancé reconnait, dans «En guise de conclusion: Le symbolique, l'imaginaire (et le réel)», que les contradictions persistent dans l'analyse car chaque ouvrage du romancier présente des éléments hétérogènes et ambivalents qui reviennent au fil de l'œuvre. On se rend vite compte que cette conclusion n'en est pas une, car Chancé non seulement semble beaucoup relativiser sa volonté classificatrice et périodisante, mais elle avance aussi à la fin une série d'autres ouvertures critiques qui mériteraient sans doute d'être approfondies, notamment le rapport entre éthique et esthétique, les notions de postcolonial et de postmoderne, le désir du Symbolique dans l'œuvre, qui laisserait envisager le retour problématique à une métaphore du père. 